\title{
Socio-demographic and behavioural correlates of oral health related quality of life among Tanzanian adults: a national pathfinder survey
}

\author{
JOYCE R. MASALU1*, EMIL N. KIKWILU1, FEBRONIA K. KAHABUKA ${ }^{1}$, MATILDA MTAYA ${ }^{1}$ and \\ AHADIELI R. SENKORO² \\ ${ }^{1}$ School of Dentistry, Muhimbili University of Health and Allied Sciences, P.O. Box 65014, Dar es Salaam, \\ Tanzania \\ ${ }^{2}$ Central Oral Health Unit, Ministry of Health, United Republic of Tanzania, P.O. Box 273, Dar es Salaam, \\ Tanzania
}

\begin{abstract}
In response to the growing recognition of quality of life measurement in health care, indicators that address the social and psychological consequences of oral disorders have been developed to complement conventional clinical assessment. The objective of this study was to determine sociodemographic and behavioural correlates of oral health related quality of life (OHRQoL) among Tanzanian adults. The national pathfinder survey methodology described in the WHO Oral Health Surveys - Basic Methods was used to obtain a total of 1,759 Tanzanian adults aged 18 years and above. In line with the pathfinder methodology clusters were purposively selected to represent cities, towns and rural areas. In each cluster individuals were stratified by age and sex. The outcome variable was the OHRQoL in terms of oral impacts on daily performances (OIDP). Frequency distribution, bivariate analyses, and generalized linear models using log binomial regression models were performed by SPSS version 15. About half (49.1\%) of the respondents reported at least one oral impact during a period of three months before the survey. Difficulty in chewing was the most prevalent impact affecting 39.9\% of participants. A higher proportion of those who reported poor conditions of teeth had difficulties in chewing and sleeping was often interrupted. Multivariate analysis indicated that those who perceived their teeth conditions to be good ( $\mathrm{PR}=0.38$; $\mathrm{CI}$ : 0.32-0.44), were of younger age ( $\mathrm{PR}=0.84$; $\mathrm{CI}$ : 0.77-0.90), and had visited a dentist during the past five years or more $(\mathrm{PR}=0.84$; $\mathrm{CI}$ : $0.77-0.90)$ and were less likely to have an oral impact. On the other hand those who restricted their sugar consumption (PR=1.12; CI: 1.030.1.22) were more likely to have an impact on at least one daily performance. It is concluded that the prevalence of OIDP was high while perceived teeth conditions and age varied with OHRQoL in terms of OIDP in the expected direction. Those who visited the dental clinic in the recent past had more impacts than those who did not. The findings of this study confirm the usefulness of subjective indicators in explaining peoples' suffering. In this regard it is recommended that in the next national pathfinder survey traditional clinical oral health indicators should be complemented with subjective indicators in order to obtain triangulated data for planning oral health services in Tanzania.
\end{abstract}

Keywords: Oral health, behaviour, oral impact, quality of life, Tanzania

\section{Introduction}

Studies have demonstrated a gap between professionally identified and self reported oral health (Onyeaso \& Arowojolu, 2003). This suggests that they document different dimensions of human experience, which are conceptually and empirically distinguished and express human suffering in distinct ways (Leao \& Sheiham, 1995). Definitions of health, disease and quality of life involve personal and social judgments that are imbued with values (Bowling, 2001). These

\footnotetext{
* Correspondence: Joyce R. Masalu; E-mail: jmasalu@muhas.ac.tz
} 
concepts are therefore constantly evolving and may vary according to social, cultural, political and practical contexts in which they are being measured.

Oral health has traditionally been measured in terms of clinical indicators. It is noted however that a restricted clinical focus emphasizing the mouth rather than the person precludes the study of the burden of oral diseases both at an individual and at the community level (Locker \& Jokovic, 1996). In response to the growing recognition of quality of life measurement in health care, indicators that address the social and psychological consequences of oral disorders have been developed to complement conventional clinical assessment. Among other socio-dental indices, Adulyanon et al., (1996) developed the Oral Impacts on Daily Performances (OIDP) inventory to be used in cross-sectional studies with an aim of discriminating between those with oral disease and those who do not. However Allen (2003) suggests further work on OHRQoL in longitudinal studies to further improve its measurements and interpretation.

Previous OIDP psychometric studies in Tanzania among young adults, (Masalu \& Astrom, 2003) and the elderly (Kida et al., 2006), demonstrated a strong association between the OHRQoL and clinical indices. Further work done in Tanzania in the recent past added evidence of discriminant validity of the OIDP applied to children and adolescents (Mtaya et al., 2007; Mashoto et al., 2009; Mbawalla et al., 2011). Although it is a common practice to use OHRQoL to distinguish individuals with clinically measured conditions from those who do not, earlier work in Tanzania (Masalu \& Astrom, 2002) indicates that perceived oral health status predicts OHRQoL as do clinical measurements. Studies indicate chewing as the frequently reported impact due to untreated oral disease and missing teeth (Masalu \& Astrom, 2002; Mtaya et al., 2007; Mashoto et al., 2009; Mbawalla et al., 2011).

Several other variables including demographic factors and level of education have been shown to predict OHRQoL (Tsakos et al., 2009). In addition level of education varies with selfreported oral pain (Ekback et al., 2010), with rural residents and those who perceive poor health conditions being more likely to report dental symptoms (Adulyanon et al., 1996). While females and younger subjects are known to be more likely to rate their oral health poorly (Locker \& Grushka, 1987); other workers (Cohen \& Jago, 1976) found sex differences varying across countries. However, Cushing et al. (1986) found no association between perceived oral health status and dental attendance.

A number of studies in Tanzania have mainly assessed oral health status by using clinical measurements in both localized studies (Rugarabamu et al., 2002; Carneiro et al., 2012) as well as in the first and second national surveys (Muya et al., 1984; Mosha et al., 2005). However psychosocial aspects related to oral health have not been assessed on a national scale. As a result of this omission, in this third national pathfinder survey the Tanzania Ministry of Health focused on psychosocial aspects of oral health. This study addressed the following research questions: Are social and behavioural factors associated with the OHRQoL? Do perceived condition of teeth and gums vary with OHRQoL in terms of OIDP? The findings will add to the growing knowledge on subjective measurements in explaining peoples' suffering and its impacts on their daily performances. The information will aid in planning oral health services in Tanzania.

\section{Materials and Methods}




\section{Selection of study sites and sampling procedure}

The national pathfinder survey methodology described in the WHO Oral Health Surveys Basic Methods (WHO, 1997) was used. The country was divided into six geographical zones. Two cities were purposively selected to represent cosmopolitan and urban clusters. From each of the remaining four zones, one region was randomly selected to provide rural clusters. In each cluster it was planned to interview 150 adults aged 18 years or more. To facilitate stratification of respondents by sex and age, each interviewer was provided with a matrix table for sex (male and female); and 5 age-groups (18-25, 26-35, 36-45, 46-55, and 56+). A total of 1759 participated in the study.

\section{Development of a questionnaire}

A pre-tested Kiswahili questionnaire was used to collect the required information. The questionnaire was pre-tested among 20 adults in each of the six administrative zones for meaning and clarity. Pre-testing was conducted in all the six zones to capture possible differences in the interpretation of words, phrases and contexts. The final version was administered twice in an interval of one week to a group of respondents from the target population but these were not included in the main survey. Reliability in terms of temporal stability showed kappa values ranging from 0.80-1.0 indicating high temporal stability of the instrument. The questionnaire was administered by six dentists who were trained on how to use the instrument so as to avoid distortion of information. No calibration of interviewers was done because there was no clinical examination. Since all interviewers were dentists we did not anticipate significant variations in reading the questions during the interviews.

\section{Measurements}

The ten items frequency scale OIDP had responses coded on a five point scale $0=$ never had any teeth problem 1 = don't know; 2 = no; 3 = sometimes; 4 = very often (appendix i). Those who never had any teeth problem were automatically coded 0 , while those who did not know if they had any difficulty were coded as " 1 "those who had problems with their teeth but the problems did not cause difficulties listed under the ten OIDP items were coded as 2. Since the weights of scores 0-2 could not be gauged in an ordinal sense; responses of each item were dichotomized yielding categories $0=$ no impact and category $1=$ perceived impacts at least sometimes (including the original score 3 - 4). Simple count scores (SC) were created for the OIDP scale by adding the 10 dummy variables. The SC OIDP scores $(0-10)$ were dichotomized, yielding categories $0=$ no daily performance affected and $1=$ at least one daily performance affected. Responses for perceived health of teeth and gums were on a six point scale: $1=$ very good, good, average, poor, and very poor to $6=$ don't know. A dichotomized variable was created with $0=$ good (including the original codes 1-2) and category $1=$ poor (including the original category $3-$ 6). Socio-demographic characteristics were assessed in terms of sex; $0=$ male and $1=$ females, place of residence; $0=$ urban and $1=$ rural, education was measured as $0=$ secondary or college education and $1=$ primary or not finished primary education. Age was assessed in years; $1=18$ 24 years, $2=25-35$ years, $3=36-45$ years and $4=46$ years and above. Dental attendance was assessed in terms of how long is it since you last visited the dental clinic? The responses were: 0 
$=$ never, less than six months ago, six to twelve months, more than a year but less than two years, two years but less than five years, to score $6=$ more than five years.

\section{Statistical analysis}

Data were analyzed using Statistical Package for Social Sciences version 15. Chi-square, Mann Whitney, Kruskal-Wallis, and Spearman rho statistics were used to assess bivariate relationships. Cronbach's alpha statistic was used to check internal consistency of the OIDP. Kappa statistic assessed temporal stability. Since the prevalence of the outcome variable i.e. OIDP was high (49.1\%) generalized linear models were used to perform multivariate analysis by log-binomial regression with statistical significance checked on the basis of $p$-value of $\leq 0.05$ and 95\% confidence intervals (CIs) for the corresponding Prevalence Ratios (PRs). All the independent variables entered in the log binomial regression analysis were significantly associated with the dependent variable (OIDP) at bivariate analysis.

\section{Ethical consideration}

Respondents were informed that they were free to choose to participate or not. Permission to interview was obtained from each individual following introduction of the interviewer by the village/street leader. Verbal consent was sought from each participating individual. Privacy was assured by interviewing one individual at time.

\section{Results}

Only 1,759 out of the targeted 2,100 adults were interviewed, giving a percent coverage of $84 \%$. Significantly higher percentages of rural than urban residents had low education, poorly rating their teeth and gingival conditions and consume alcohol. Whereas significantly lower proportions of rural than urban residents reported to consume sugared snacks and had visited a dental clinic in less than five years before the survey (Table 1).

Table 1: Distribution of subjects by socio-demographic and behavioural characteristics by residence ( $N=1759$, number in parenthesis)

\begin{tabular}{|l|c|l|l|}
\hline Socio-demographic characteristics & Response & Urban & Rural \\
\hline Age & $18-24$ & $25.7(188)$ & $20.5(211)^{\mathrm{ns}}$ \\
\hline & $26-35$ & $22.8(167)$ & $24.4(251)$ \\
\hline & $36-45$ & $19.8(145)$ & $20.5(211)$ \\
\hline Sex & $46+$ & $31.7(232)$ & $34.5(354)$ \\
\hline & Females & $49.5(362)$ & $52.0(534)^{\mathrm{ns}}$ \\
\hline Education & Males & $50.5(370)$ & $48.0(493)$ \\
\hline & High & $28.8(211)$ & $80(82)^{* * *}$ \\
\hline Perceived teeth condition & Low & $71.2(521)$ & $92.0(945)$ \\
\hline & Good & $49.0(359)$ & $36.7(377)^{* * *}$ \\
\hline Perceived gingival condition & Poor & $51.0(373)$ & $63.3(650)$ \\
\hline & Good & $62.8(460)$ & $49.5(508)^{* * *}$ \\
\hline Visit dentist & Poor & $37.2(272)$ & $50.5(519)$ \\
\hline
\end{tabular}




\begin{tabular}{|l|l|l|l|}
\hline & Less than 5 years & $34.8(255)$ & $24.6(253)$ \\
\hline & Rare or never & $26.9(197)$ & $61.4(631)^{* * *}$ \\
\hline Use sugared snacks & $\begin{array}{l}\text { At least once a } \\
\text { week }\end{array}$ & $73.1(535)$ & $38.6(396)$ \\
\hline Alcohol use & \multicolumn{1}{|c|}{ No } & $66.4(486)$ & $58.1(597)^{* * *}$ \\
\hline & \multicolumn{1}{|c|}{ Yes } & $33.6(246)$ & $41.9(430)$ \\
\hline
\end{tabular}

Chi-square test: ${ }^{* * *}=\mathrm{p}<0.001$, ns $=$ not significant

The ten OIDP items had an inter-item reliability coefficient (Chronbach's alpha of 0.99). All the OIDP items were highly correlated with Spearman's rho coefficients ranging from 0.86 to 0.97 at $P<0.001$. Distribution of OIDP frequency items revealed that impact on eating was reported to be the most prevalent impairment among urban (34.6\%) and rural residents $(42.1 \%)$. The second frequently reported impact was interrupted sleep in both urban (28.8\%) and rural (37.6\%) residents. Overall, $49.1 \%$ of the study participants reported at least one impact during the previous three months (Table 2).

Table 2: Oral Impacts on Daily Performance according to residence (N=1759, number in parenthesis)

\begin{tabular}{llll}
\hline OIDP performance items & Urban & Rural & Total \\
\hline Difficult in chewing & $34.6(253)$ & $42.1(432)^{* *}$ & $38.9(685)$ \\
Difficulty with speech & $9.6(70)$ & $9.0(92)^{\mathrm{ns}}$ & $9.2(162)$ \\
Embarrassed by teeth appearance & $6.7(49)$ & $4.8(49)^{\mathrm{ns}}$ & $5.6(98)$ \\
Felt tense because teeth/mouth & $13.3(97)$ & $16.2(166)^{\mathrm{ns}}$ & $15.0(263)$ \\
Avoid smiling & $8.1(59)$ & $6.3(65)^{\mathrm{ns}}$ & $7.0(124)$ \\
Sleep interrupted & $28.8(211)$ & $37.6(386)^{* * *}$ & $33.9(597)$ \\
Days taken off work & $14.2(104)$ & $16.6(170)^{\mathrm{ns}}$ & $15.6(274)$ \\
Difficulty doing usual activities & $13.0(95)$ & $14.4(148)^{\mathrm{ns}}$ & $13.8(243)$ \\
Less tolerant of spouse or people & $6.1(45)$ & $3.3(34)^{\mathrm{ns}}$ & $4.5(79)$ \\
Reduced social activities & $9.6(70)$ & $7.9(81)^{*}$ & $8.6(151)$ \\
Overall impact & $43.2(316)$ & $53.3(547)^{* * *}$ & $49.1(863)$ \\
\hline
\end{tabular}

Chi-square test: ${ }^{*} p<0.05,{ }^{* *} p<0.01^{* * *} p<0.001,{ }^{n s}=$ not significant

Significantly higher proportions of individuals who perceived poor conditions of teeth or gums reported impacts for all the ten items than those who rated their teeth or gums to be in good conditions. All the differences were significant at $\mathrm{p}<0.001$ (Table 3).

Table 3: Occurrence of Oral Impacts on Daily Performance according to perceived status of teeth and gums ( $=1759$, number in parenthesis)

\begin{tabular}{lllll}
\hline OIDP performance items & \multicolumn{2}{l}{ Perceived teeth status } & \multicolumn{2}{l}{ Perceived teeth gingival status } \\
& Good & Poor & Good & Poor \\
\hline Difficulty in chewing/biting & $16.0(118)$ & $55.4(567)$ & $28.5(276)$ & $51.7(409)$ \\
Difficulty with speech & $2.2(16)$ & $14.3(146)$ & $6.1(59)$ & $13.0(103)$ \\
Embarrassed about teeth & $1.2(9)$ & $8.7(89)$ & $2.8(27)$ & $9.0(71)$ \\
Feel tense & $3.8(28)$ & $23 .(235)$ & $9.0(87)$ & $22.3(176)$ \\
Avoid smiling & $1.8(13)$ & $10.9(111)$ & $3.0(29)$ & $12.0(95)$ \\
Sleep interrupted & $14.3(105)$ & $48.1(492)$ & $25.1(243)$ & $44.8(354)$
\end{tabular}


Days taken off work

Doing usual activities

Less tolerant of spouse

Reduced social activities

Overall impact
3.5 (26)

$2.2(16)$

$0.7(5)$

$1.2(9)$

$22.1(163)$
$24.2(248)$

$22.2(227)$

$7.2(74)$

$13.99142)$

$68.4(700)$
$9.5(92)$

$8.5(82)$

$2.8(27)$

$4.6(45)$

37.1 (359)
$23.0(182)$

$20.4(161)$

$6.6(52)$

$13.4(106)$

$63.7(504)$

Chi-square test: all differences between groups were statistically at $\mathrm{p}<0.001$

Bivariate associations between the OIPD (SC) and the independent variables using Mann Whitney and Kruskal-Wallis tests indicated statistically significant higher OIDP (SC). Mean Ranks among females, those with old age, perceiving teeth and gums being poor, low education, took alcohol, restricted sugar intake, reside in rural area and visited a dentist within less than five years than their corresponding counterparts. Controlling for background variables, those who perceived their teeth conditions to be good were less likely to have an impact on their daily performance ( $P R=0.38$; $C I$ : 0.32-0.44), while those who visited the dentist five years ago or more were less likely to have an impact ( $\mathrm{PR}=0.84$; $\mathrm{CI}$ : 0.77-0.90). Those who rarely consumed sugar were more likely to have an impact ( $P R=1.12 ; C I$ : 1.03-0.1.22). As compared to older individuals ( $\geq 46$ years), the youngest ones (18-24 years) were less likely to have an impact (Table 4). Sex, residence, alcohol consumption, education and gingival condition did not have significant associations with OIDP in the multivariate analysis.

Table 4: PRs and 95\% CI for respondents Oral Impacts on Daily Performance

\begin{tabular}{ll}
\hline Factors associated with OIDP & PR $\mathbf{( 9 5 \%} \mathbf{C I})$ \\
\hline Male $=0$ & $0.98(0.91-1.05)$ \\
Female 1 & 1 \\
18-24 years & $0.83(0.72-0.94)^{*}$ \\
26-35 years & $0.98(0.89-1.07)$ \\
$36-45$ years & $0.99(0.90-1.09)$ \\
$46+$ years & 1 \\
Urban $=0$ & $0.95(0.87-1.04)$ \\
Rural $=1$ & 1 \\
High education $=0$ & $0.94(0.83-1.07)$ \\
Low education $=1$ & 1 \\
Did not take alcohol $=0$ & $0.94(0.87-1.01)$ \\
Took alcohol $=1)$ & 1 \\
No sugared snacks $=0$ & $1.12(1.02-1.22)^{*}$ \\
Eat sugared snacks $=1$ & 1 \\
Visited a dentist $5^{+}$yrs or never $=0$ & $0.84(0.77-0.90)^{* * *}$ \\
Visited a dentist $\leq 5$ yrs $=1$ & 1 \\
Perceived good teeth $=0$ & $0.38(0.32-0.44)^{* * *}$ \\
Perceived poor teeth $=1$ & 1 \\
Perceived good gums $=0$ & $0.93(0.86-1.01)$ \\
Perceived poor gums $=1$ & 1 \\
\hline
\end{tabular}

Log Binomial regression with OIDP as dependent variable: ${ }^{*} p<0.05,{ }^{* *} p<0.01^{* * *} p<0.001$ 


\section{Discussion}

This paper documents the associations between OHRQoL and socio-behavioural factors and self rated teeth and gingival conditions among Tanzanian adults. This is the first Tanzanian study on OHRQoL with almost a national coverage which offers national baseline information of these subjective measurements. This study used the WHO Basic methods - oral health surveys pathfinder methodology (WHO, 1997) which is widely used in dentistry especially when the information collected is for planning oral health services. However this method is less rigorous in picking very representative samples (WHO, 1997).

In this study the modified OIDP version used among Tanzanian adults clearly distinguished those with perceived teeth problems from those who did not. Similar findings have been reported in previous studies conducted in children, youths and elderly Tanzanians as well as among adolescents in Uganda (Masalu et al., 2003; Astrom \& Okullo, 2003; Kida et al., 2006, Mtaya et al., 2007; Mashoto et al., 2009; Mbawalla, 2011). These findings add evidence to earlier work which support the use of subjective indicators to describe human suffering (Locker \& Jokovic, 1996). With the findings of this national sample and those of previous studies which targeted localised sites in Tanzania, it suffices to suggest that the OIDP index is applicable to the Tanzania context. These finding are likely to be useful in addressing inequity in oral health by focusing attention to those segments of the population that reported more impacts due to poor teeth conditions.

About half of the participants had at least one oral impact because of poor state of their teeth. This proportion is lower as compared to $73.6 \%$ that was reported by Adulyanon et al. (1996) among Thai adult population. This difference could be attributed to differences in age groups used in the two studies. In addition, cultural differences have been shown to influence perception of impacts elsewhere (Tsakos et al., 2001). Chewing difficulties was the most predominant impact in this study. Similar findings have been reported in other studies conducted in Tanzania (Masalu \& Astrom, 2002; Mtaya et al., 2007; Mashoto et al., 2009). To most individuals in both developing (Benoit et al., 2005; van Palenstein \& Nathoo, 1990) and developed countries (Locker \& Grushka, 1987), difficulties in chewing are mainly due to pain from untreated dental disease. As clearly stipulated in the ninth strategic objective of Tanzania policy guidelines for oral health care, preventive and emergency oral health services have to be equitably distributed to the majority of Tanzanians to reduce unnecessary suffering due to toothache (MOH, 2002).

Relatively small proportions of study participants reported to feel embarrassed, avoid smiling or felt their spouses becoming less tolerant due to dental problems, with higher percentage of rural than urban residents rating their teeth and gingival conditions to be poor. A smaller proportion of rural than urban residents in this study visited dental clinics within the previous five years because most dental clinics in Tanzania are located in urban areas. Generally oral pain is prevalent among Tanzanians but a small proportion would seek dental care (Kikwilu et al., 2008). The fact that rural residents constitute the bulk of the work force in Tanzania, extension of preventive and emergency dental services to remote rural Tanzania should be seen as a requirement for economic growth in Tanzania. 
Expectedly those who reported poor teeth condition had impacts for most OIDP items. However perceived poor gingival conditions were not significantly associated with oral impacts in the multivariate analysis. Studies conducted in Tanzania on periodontal diseases indicate high prevalence of gingival inflammation and calculus accumulation. However periodontal breakdown is minimal (Bealum et al., 1986; Mumghamba et al., 2006). Naturally in the absence of active pain from gingival conditions the problems tend to occur without causing much concern to those affected. Moreover the commonest cause of tooth loss in Tanzania is dental caries (Mosha \& Lema, 1991). Making people worry about dental caries because of its squeal on tooth loss.

Age varied with OIDP in the expected direction due to cumulative nature of dental caries experience, with the elderly having more impacts than the young ones. Individuals who reported to have visited a dental clinic in the recent past were more likely to be those who had impacts. Although distance to health facility and cost are important barriers for most Tanzanians to access dental treatment (Kikwilu et al., 2008), unbearable pain leave people with no choice but to seek care for pain relief. These visits are unlikely to be for a simple dental check up and this might explain why those who visited the clinics were more likely to have impacts. Ironically those who reported a restricted sugar intake were more likely to report impacts. Nevertheless this being a cross-sectional study where both cause and outcome are measured at one point in time the most logical explanation would be the already aching teeth become even more painful upon contact with sugar and force those affected to refrain from taking sugared foods and drinks.

This study has a number of limitations. Lack of information about the nonrespondents precludes any conclusion about a possible selection bias, although the percent coverage was high enough to assume that the target population is reflected to a reasonable degree. The clusters and strata were purposively selected to capture the diversity of characteristics as directed in the pathfinder methodology. However the individuals were let to participate conveniently until the quota size was attained for each cluster and strata. Therefore it is difficult to work out the probability of an individual to be selected in the sample. To this effect it is not possible to adjust for the design effect and volunteer bias cannot be overruled. Therefore the findings must be interpreted with caution when making direct generalizations to the whole country. Nevertheless the pre-stratification by age and sex in specified quotas might have redressed the bias to some extent. The present study relied on self reported information; a possibility of over and under reporting due to recall and social desirability biases exists. Lack of back-translation of the modified OIDP version may also be a drawback of this study. Furthermore, at the point of analysis some ordinal and continuous variables were transformed to allow cross tabulation and calculation of PRs. The data transformation might have placed individuals into wrong categories and reduced the power of the study.

The modified OIDP hereby employed to assess OHRQoL in a national sample has provided a quick and relevant glint of what people feel about their oral health and how the whole scenario impacts on the quality of their lives. In conclusion, the findings of this study indicate that the prevalence of OIDP was $49.1 \%$ while perceived teeth conditions and age varied with OHRQoL in terms of OIDP in the expected direction. Those who visited the dental clinic in the recent past had more impacts than those who did not. The findings of this study confirm the 
usefulness of subjective indicators in explaining peoples' suffering. In this regard it is recommended that in the next national pathfinder survey; traditional clinical oral health indicators should be complemented with subjective indicators in order to obtain triangulated data for planning oral health services in Tanzania.

\section{Competing interests}

The authors declare that they have no competing interests.

\section{Authors' contributions}

JRM participated in conception and design of study, data collection, analysis, interpretation and writing of the manuscript. EK participated in the design of the study, data collection and revision of the manuscript. FK participated in development of the proposal, data collection, and revision of the manuscript. MM participated in the writing of the manuscript. AS formulated the research question, participated in data acquisition and reviewed the manuscript

\section{Acknowledgements}

The authors thank the Ministry of Health and Social Welfare for funding and embracing the psychosocial approach to oral health in its third national pathfinder survey. Special thanks to Professor Paul Eric Petersen for providing a draft of the simplified oral health questionnaire for adults.

\section{References}

Adulyanon S., Vourapukjaru J., \& Sheiham A. (1996) Oral impact affecting daily performances in a low dental disease Thai population. Community Dentistry and Oral Epidemiology 24, $385-389$.

Allen P.F. (2003) Assessment of oral health related quality of life. Health Quality of Life Outcomes 1:40.

Astrom, A.N., \& Okullo, I. (2003) Validity and reliability of oral impacts on daily performance OIDP frequency scale: a cross-sectional study of adolescents in Uganda. Biomed Central Oral Health 3:5.

Bowling A. (2001) Measuring disease; a review of disease specific quality of life measurement scales. Open University Press, Second Edition, Chapter 1 Pg 14-35

Benoit, V., Msellati, P., Zoungrana, C., Fournet, F. \& Salem, G. (2005) Reasons for attending dental care services in Oagadougou, Burkina Faso. Bulletin of the World Health Organization 83, 9.

Carneiro, L.C. \& Kabulwa, M.N. (2012) Dental caries, and supra-gingival plaque and calculus among students in Tanga, Tanzania ISRN Dent 245296.

Cohen, L.K. \& Jago, J.D. (1976) Towards the formulation of social dental indicators. International Journal of Health Service 6, 681-698. 
Cushing, S., Sheiham, A. \& Maizel, J. (1986) Developing socio-dental indicators - the social impact of dental diseases. Community Dental Health 3, 3-17.

Ekback, G., Astrom, N.A., Ordell, K.S. \& Unell, L. (2010) Satisfaction with teeth and life-course predictors; a prospective study of a Swedish 1942 birth cohort. European Journal of Oral Science 118, 66-74.

Kida, I.A., Astrom, A.N., Strand, G.V., Masalu, J.R. \& Tsakos, G. (2006) Psychometric properties and the prevalence, intensity and causes of oral impacts on daily performance (OIDP) in a population of older Tanzanians. Health Quality of Life Outcomes 4, 56.

Kikwilu, E.N., Masalu, J.R., Kahabuka, F.K. \& Senkoro, A.R. (2008) Prevalence of oral pain and barriers to use of emergency oral health care facilities among adult Tanzanians. BMC Oral Health 29, 28.

Kikwilu, E.N., Kahabuka, F.K., Masalu, J.R. \& Senkoro, A.R. (2009) Satisfaction with urgent oral care among adult Tanzanians. Journal of Oral Science 51, 47-54.

Leao, A. \& Sheiham, A. (1995) Relation between clinical dental status and subjective impacts on daily living. Journal of Dental Research 1408-1413.

Locker, D. \& Grushka, M. (1987) The impact of oral and facial pain. Journal of Dental Research 66, $1414-1417$

Locker, D. \& Jokovic, A. (1996) Using subjective oral health status indicators to screen for dental care in older adults. Community Dentistry and Oral Epidemiology 24, 398-402.

Masalu, J.R. \& Astrom, A.N., (2002) Social and behavioural correlates of oral quality of life studied among university students in Tanzania. Acta Odontologica Scandinavica 60, 353359.

Masalu, J.R. \& Astrom, A.N. (2003) Applicability of an abbreviated version of the oral impacts on daily performances (OIDP) scale for the use among Tanzanian students. Community Dentistry and Oral Epidemiology 31, 7-14.

Masalu, J.R., Kikwilu, E.N., Kahabuka, F.K., Senkoro, A.R. \& Kida, I.A. (2009) Oral health related behaviors among Tanzanian adults: A national pathfinder survey. BMC Oral Health 9:22.

Mashoto, K.O., Astrom, A.N., David, J. \& Masalu, J.R. (2009) Dental pain, oral impacts and perceived need for dental treatment in Tanzanian school students: a cross-sectional study. Health Quality of Life outcomes 7:73.

Mbawalla, H.S., Mtaya, M., Masalu, J.R., Brudvik, P. \& Asrom, A.N. (2011) Discriminative ability of the generic and condition specific child-oral impacts on daily performances (Child-OIDP) by the Limpopo-Arusha School Health (LASH) project: a cross-sectional study. BMC Pediatrics 11:45.

MOH (2002) Policy Guidelines for Oral Health Care in Tanzania. Ministry of Health, United Republic of Tanzania, Dar es Salaam 12 pp.

Mosha, H.J., Senkoro, A.R., Masalu, J.R.P., Kahabuka, F., Mandari, G., Mabelya, L. \& Kalyanyama, B., (2005) Oral health status and treatment needs among Tanzanians of different age groups. Tanzania Dental Journal 12, 18-27.

Mosha, H.J. \& Lema, P.A. (1991) Reasons for tooth extraction among Tanzanians. East African Medical Journal 68, 10-14. 
Mtaya, M., Astrom, A.N. \& \& Tsakos, G. (2007) Applicability of an abbreviated of the Child OIDP inventory among primary schoolchildren in Tanzania. Health and Quality of Life Outcomes 5:40.

Mumghamba, E.G, Manji, K.P. \& Michael, J. (2006) Oral hygiene practices, periodontal conditions, dentition status and self reported bad mouth young mothers, Tanzania. International Journal of Dental Hygiene 4, 166 -173.

Muya, R., Rambusch, E., Fejerskov, O., Hobdell, M.H. \& Normark, S. (1984) Changing and Developing Dental Health Services in Tanzania 1980-2000. Dar es Salaam, Tanzania: Ministry of Health and DANIDA.

Onyeaso, C.O. \& Arowojolu, M.O. (2003) Perceived, desired and normatively determined orthodontic treatment needs among orthodontically untreated Nigerian adolescents. West African Journal of Medicine 22, 5-9.

Rugarabamu, P.G.N., Poulsen, S. \& Massalu, J.R. (2002) A longitudinal study of occlusal caries among school children in Dar es Salaam Tanzania. Community Dentistry and Oral Epidemiology 30, 47-51.

Tsakos, G., Marcenes, W. \& Sheiham, A. (2001) Cross cultural differences in oral impacts on daily performance between Greek and British older adults. Community Dental Health 18, 209-213.

Tsakos, G., Sheiham, A., Iliffe, S., Kharicha, K., Harari, D., Swift, C.G., Gillman, G. \& Stuck, A.E. (2009) The impact of educational level on oral health related- quality of life in older people living in London. European Journal of Oral Sciences 117, 286-292.

van Palenstein, W.H. \& Nathoo, Z.A. (1990) Dental treatment demands among patients in Tanzania. Community Dentistry and Oral Epidemiology 18, 85-87.

WHO (1997) Oral Health Surveys, Basic Methods 4. World Health Organization, pp 5-9. 